\title{
Zeeman Effect Observed in the Evanescent Wave
}

\author{
T. Kawalec, M.J. Kasprowicz, L. Józefowski \\ AND T. DOHNALIK \\ Smoluchowski Institute of Physics, Jagiellonian University \\ Reymonta 4, 30-059 Cracow, Poland
}

(Received December 8, 2003; in final form March 5, 2004)

We have observed a Zeeman effect in an evanescent wave absorption in $\mathrm{Rb}$ vapor with a single-mode tunable diode laser. The spectra are obtained for several magnetic field- and laser light-configurations enabling the observation of different $\pi$ and $\sigma$ Zeeman split lines contribution. The relative amplitudes and relative frequencies of all Zeeman components were calculated theoretically and compared with measured spectra. The analysis of the data can lead to the determination of the polarization state of the evanescent wave.

PACS numbers: 42.25.Gy, 32.60.+i

\section{Introduction}

When a light beam enters the boundary layer of two dielectrics over the critical angle the total internal reflection occurs. However, the light penetrates the dilute medium creating the evanescent wave. Its penetration depth is of the order of the wavelength. When the dilute medium is an atomic or molecular gas one can talk about the evanescent wave spectroscopy (EWS) [1]. EWS deals with atoms or molecules in a very small volume lying very close to the surface at the boundary. With applied magnetic field $B$ it is easy to obtain a high magnitude and great homogeneity of $B$ in the evanescent wave region. In the paper we present the Zeeman effect in the $\mathrm{Rb}$ vapor observed in the evanescent wave in different geometrical configurations. It is a primary step to the investigation of the effective polarization of the evanescent wave. 


\section{Theory}

It is well known that the total internal reflection occurs if the angle of incidence $\theta$ is greater than $\theta_{c}=\arcsin n_{2} / n_{1}$, where $n_{1}>n_{2}$ (Fig. 1 ). The angle of refraction becomes imaginary and the evanescent wave is described by the wave number $k$ with the complex $z$ component [2]:

$$
\begin{aligned}
& k_{x}=k_{0} n_{1} \sin \theta \\
& k_{z}=i k_{0} n_{2} \sqrt{\left(\frac{n_{1}}{n_{2}} \sin \theta\right)^{2}-1,}
\end{aligned}
$$

where $k_{0}$ is the wavenumber in vacuum. Substituting Eq. (1b) into the wavefunction of the electric field $\boldsymbol{E}=\boldsymbol{E}_{0} \exp [\mathrm{i}(\boldsymbol{k} \cdot \boldsymbol{r}-\omega t)]$ one gets the damping factor $\exp \left(-\left|k_{z}\right| z\right)$. The quantity $\left|k_{z}\right|^{-1}$ is interpreted as a penetration depth and for angles of incidence close to the critical angle it is of the order of the wavelength. The Goos-Hänchen shift occurs in the OX direction, which is represented by the quantity $x_{s}$ in Fig. $1[3,4]$.

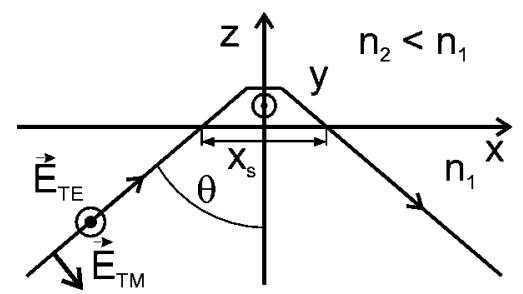

Fig. 1. The scheme of the total internal reflection.

In the experiment we measure the quantity $R$, where $R=|r|^{2}$ is the reflection coefficient obtained from the Fresnel equations for the TM and TE polarization of the incident wave $\left(n_{21}=n_{2} / n_{1}\right)$ :

$$
\begin{aligned}
& r_{\mathrm{TM}}=\frac{n_{21}^{2} \cos \theta-\sqrt{n_{21}^{2}-\sin ^{2} \theta}}{n_{21}^{2} \cos \theta+\sqrt{n_{21}^{2}-\sin ^{2} \theta}} \\
& r_{\mathrm{TE}}=\frac{\cos \theta-\sqrt{n_{21}^{2}-\sin ^{2} \theta}}{\cos \theta+\sqrt{n_{21}^{2}-\sin ^{2} \theta}} .
\end{aligned}
$$

For the complex index of refraction $\mathrm{n}_{2} \approx 1+\mathrm{i} \kappa(\kappa \ll 1)$ we get:

$$
\begin{aligned}
& R_{\mathrm{TM}} \approx 1+\left(2 n_{1}^{2} \sin ^{2} \theta-1\right) \frac{4 n_{1} \kappa \cos \theta}{\left[\cos ^{2} \theta+n_{1}^{2}\left(n_{1}^{2} \sin ^{2} \theta-1\right)\right]} \\
& R_{\mathrm{TE}} \approx 1+\frac{4 n_{1} \kappa \cos \theta}{\left[n_{1}^{2} \cos ^{2} \theta+\left(n_{1}^{2} \sin ^{2} \theta-1\right)\right]}
\end{aligned}
$$


where $\kappa$ describes the absorption by the atomic vapor in the vicinity of the dielectric interface and is proportional to the square of the matrix element of the electric dipole operator for a given transition.

The strengths and positions of the lines in the absorption spectra of Rb were found as the functions of a magnitude of an external magnetic field [5]. In the field we used (of the order of $0.1 \mathrm{~T}$ ), the quantum numbers $\boldsymbol{F}=\boldsymbol{I}+\boldsymbol{J}$ are not conserved because of the mixing of the magnetic sublevels with the same numbers $M$, but with different numbers $F$. In the Schrödinger equation

$$
H_{\mathrm{at}}\left|L_{F}, M\right\rangle=E_{L_{F} M}\left|L_{F}, M\right\rangle
$$

we have taken into account quantum numbers $L_{F}$, where $L_{F} \rightarrow F$ when $B \rightarrow 0$. The subscript denotes number $F$ of the particular level in the zero magnetic field (see Figs. 2 and 3 ). $H_{\text {at }}$ is the atomic Hamiltonian and $E_{L_{F} M}$ is the energy of the

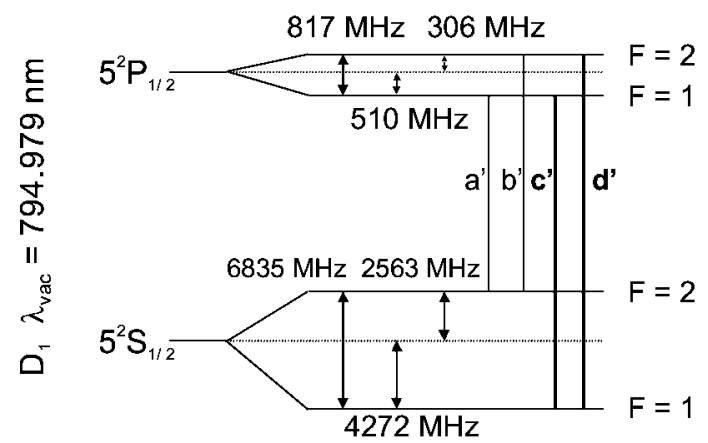

Fig. 2. Hyperfine structure of the ground and first excited state of $R b^{87}$ atoms (after $[6,7])$.

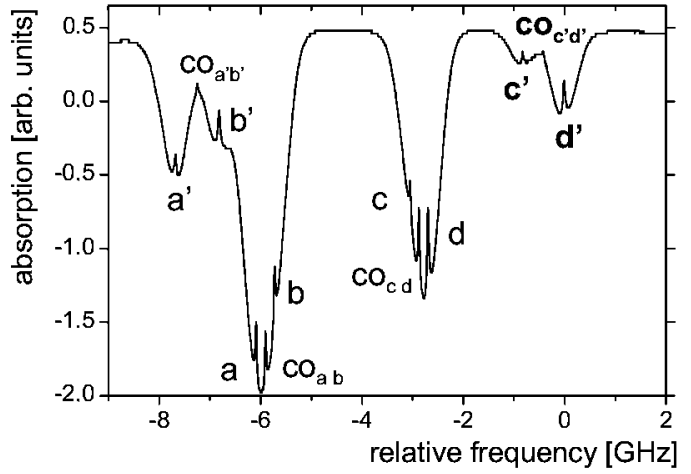

Fig. 3. Saturated spectrum of $\mathrm{Rb}^{87}\left(I=3 / 2\right.$; lines $a^{\prime}$ to $\left.d^{\prime}\right)$ and $\mathrm{Rb}^{8.5}(I=5 / 2$; lines $a$ to $d)$. The cross-over resonances are shown $(c o)$. 
$L_{F}$ level. One can represent the atomic state as the sum of $|F, M\rangle$ states:

$$
\left|L_{F}, M\right\rangle=\sum_{F} C_{F M}^{L_{F}}|F, M\rangle
$$

where

$$
\sum_{F}\left|C_{F M}^{L_{F}}\right|^{2}=1
$$

The calculations of the line strengths and energies were performed for both $\pi$ and $\sigma$ components of the $c^{\prime}$ and $d^{\prime}$ transitions (see Fig. 2 and the calculated curves in Fig. 6).

\section{Experimental setup}

The experimental setup consisted of a cylinder-shaped quartz cell with a reservoir heated to $433 \pm 2 \mathrm{~K}$, magnetic field, the reference saturation spectroscopy arrangement and the Fabry-Pérot interferometer. Two last ones allowed us to calculate the current frequency of the laser radiation. The exemplary saturation spectroscopy spectrum is shown in Fig. 3. The cell was $30 \mathrm{~mm}$ in diameter and $50 \mathrm{~mm}$ high and contained natural mixture of $\mathrm{Rb}^{85}$ and $\mathrm{Rb}^{87}$ vapor at the density of $2 \times 10^{14} \mathrm{~cm}^{-3}$ (which corresponds to the pressure of $1.33 \mathrm{~Pa}$ [8]). One of the bases of the cell was common to the base of the quartz prism with refractive index $n=1.45$ (see Fig. 4)

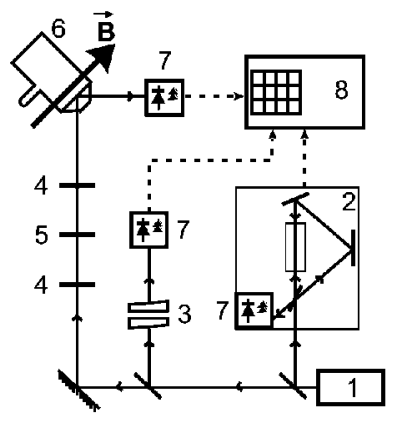

Fig. 4. Experimental setup: 1 - laser, 2 - saturation spectroscopy setup, 3 - FP interferometer, 4 - polarizer, 5 - half-waveplate, 6 - oven with quartz cell, 7 photodiodes, 8 - data acquisition system.

We used two hyperfine components of the rubidium $D_{1}$ line: $F=1 \rightarrow F=$ $1\left(c^{\prime}\right)$ and $F=1 \rightarrow F=2\left(d^{\prime}\right)$ at $\lambda=794.98 \mathrm{~nm}$ (see Figs. 2, 3). The magnitude of the applied magnetic field was controlled by the proper setting of the distance between two constant magnets. The value of $B(0.08$ to $0.15 \mathrm{~T})$ allowed us to resolve some of the Zeeman components of the Doppler-broadened spectrum. A laser beam (with the light intensity of $1 \mu \mathrm{W} / \mathrm{mm}^{2}$ ) came from the EOSI 2001 diode 

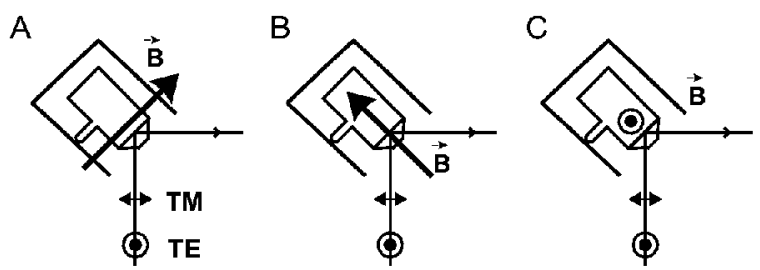

Fig. 5. Configurations $\mathrm{A}, \mathrm{B}$, and $\mathrm{C}$ of the magnetic field.

laser with a DMD795 head and tunable external resonator. The laser linewidth was less than $1 \mathrm{MHz}$. We used different orientations of the magnetic field and two linear polarizations of the incident beam (parallel and perpendicular to the plane of incidence) denoted as TM and TE (see Fig. 5).

\section{Results}

The obtained spectra for different polarizations of the incident beam and for different configurations A, B, and C are shown in Figs. 6-10, where the origin of the relative frequency scales corresponds to the $d$ 'transition. The angle of incidence was set to about $2 \mathrm{mrad}$ over the critical angle. In Fig. $6 \mathrm{a}$ and b there are shown two exemplary spectra with the fitted functions. The fitting procedure served only for finding the exact magnitude of $B$ in the evanescent wave volume and for normalizing the experimental and calculated profiles. For simplicity the theoretical function consisted of Gaussian curves. The value of $B$ was treated as a parameter in the amplitudes and positions of the Gaussian profiles obtained by solving Eq. (4). The calculated magnitude of the magnetic field was $0.102 \pm$ $0.001 \mathrm{~T}$ for each presented spectrum. The agreement between the theoretical and

a)

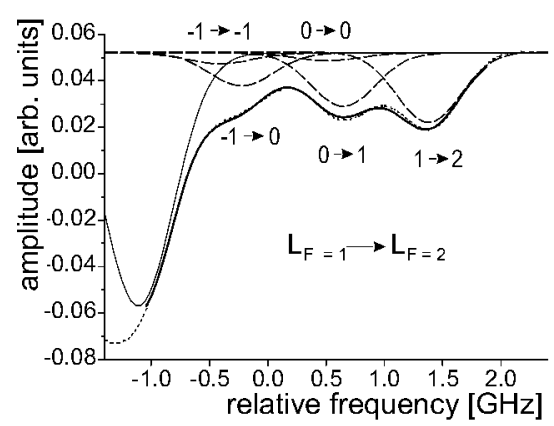

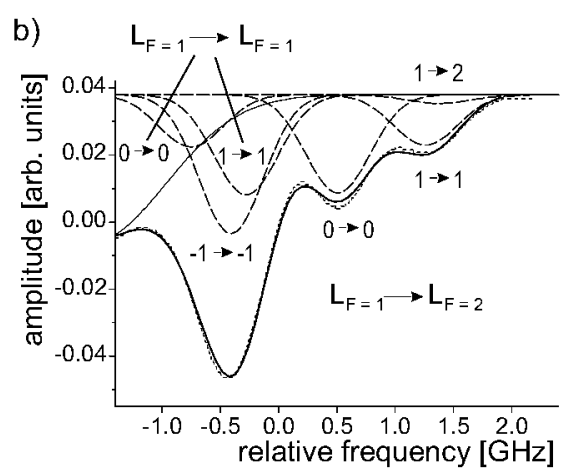

Fig. 6. Two exemplary spectra (dotted lines) with some of the Gaussian profiles (dashed lines) as the parts of the calculated profiles (solid lines): polarization TM in the configuration $\mathrm{C}(\mathrm{a})$ and polarization TM in the configuration B (b). The thin solid line represents the influence of $R b^{85}$. 
experimental spectra shows that the polarization of the evanescent wave could be described by the base polarizations $\pi$ and $\sigma$.

In Figs. 7-10 one can see partly resolved lines corresponding to the Zeeman splitting. The curves are different for $\pi$ and $\sigma$ lines in the Zeeman effect. In Fig. 7 we present the signals obtained for different configurations but always with the

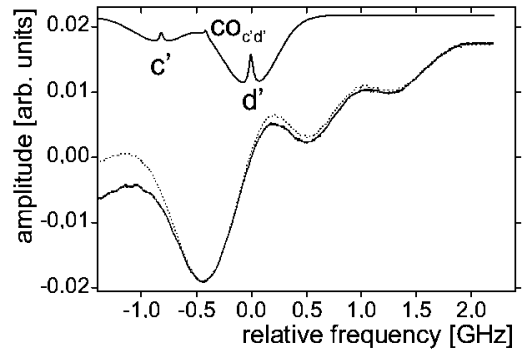

Fig. 7. The spectra for the polarization TE in the configuration $\mathrm{C}$ (solid line) and for the polarization TM in the configuration B (dotted line); polarization of the evanescent wave is $\pi$ in both cases. The saturation spectroscopy spectrum is shown as a reference (see also Fig. 3).

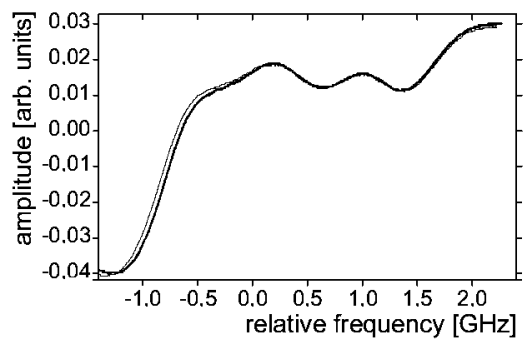

Fig. 8. The spectra for the polarization TE in the configuration A (thick solid line) and for the polarization TE in the configuration B (thin solid line); polarization of the evanescent wave is $\sigma$ in both cases.

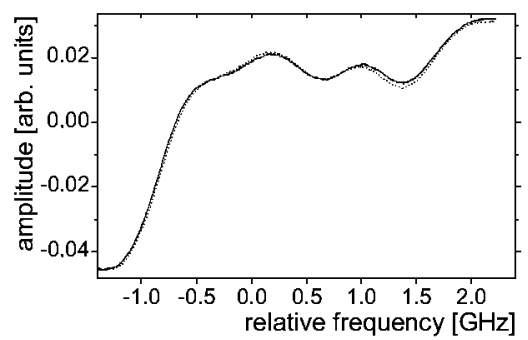

Fig. 9. The spectra for the polarization TE in the configuration B (solid line) and for the polarization TM in the configuration $\mathrm{C}$ (dotted line); polarization of the evanescent wave is $\sigma$ in both cases. 


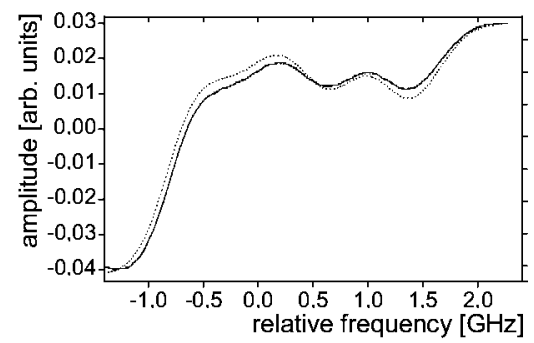

Fig. 10. The spectra for the polarization TE in the configuration A (solid line) and for the polarization TM in the configuration A (dotted line); polarization of the evanescent wave is $\sigma$ in both cases.

light polarization parallel to the magnetic field. In Fig. 8 we present the signals for different configurations but always with the light polarization perpendicular to the magnetic field. In Figs. 9 and 10 for comparison we present the signals obtained for different polarizations but with the same angle to the magnetic field. The curves in Figs. 7 and 10 do not overlap which could be partly due to a not perfect polarization of the laser beam, which is about $50: 1$ only.

\section{Conclusions}

We checked the possibility of observing the Zeeman effect for the atoms interacting with the evanescent wave. It looks very interesting because of the very small volume of the interaction, which helps to obtain high, homogeneous magnetic field. The proximity of the surface enables the investigation of the surface-atom interactions.

The other interesting aspect might be the investigation of the evanescent wave polarization which is expected to be not a pure linear TE one [9]. The quantitative analysis of that polarization state will be done later on after completing other experiments using e.g. an optogalvanic effect.

\section{References}

[1] G. Nienhuis, F. Schuller, M. Ducloy, Phys. Rev. A 38, 5197 (1988).

[2] M. Born, E. Wolf, Principles of Optics, 5th ed., Pergamon Press, New York 1975.

[3] F. Goos, H. Hänchen, Ann. Phys. (Leipzig) 1, 333 (1947).

[4] E. Pfleghaar, A. Marseille, A. Weis, Phys. Rev. Lett. 70, 2281 (1993).

[5] A. Messiah, Mécanique Quantique, Vol. 2, Dunod, Paris 1964.

[6] G.P. Barwood, P. Gill, W.R.C. Rowley, Appl. Phys. B 53, 142 (1991).

[7] S. Bize, Y. Sortais, M.S. Santos, C. Mandache, A. Clairon, C. Salomon, Europhys. Lett. 45, 558 (1999).

[8] A.N. Nesmeyanov, Vapor Pressure of the Chemical Elements, Elsevier, Amsterdam 1963.

[9] F. de Fornel, Evanescent Waves, Springer, Berlin 2001. 